\title{
Fractional Fourier Series with Separation of Variables Technique and Its Application on Fractional differential Equations.
}

\author{
AHMED BOUCHENAK \\ University of Jordan \\ Department of Mathematics \\ Amman \\ Jordan \\ bouchenakmath@gmail.com
}

\author{
KHALIL ROSHDI \\ University of Jordan \\ Department of Mathematics \\ Amman \\ Jordan \\ roshdi@ju.edu.jo
}

\author{
ALHORANI MOHAMMED \\ University of Jordan \\ Department of Mathematics \\ Amman \\ Jordan \\ horani@ju.edu.jo
}

Abstract: When using some classical methods, such us separation of variables; it is impossible to find a general solution for some differential equations. Therefore, we suggest adding conformable fractional Fourier series to get a new technique to solve fractional Benjamin Bana Mahony and Heat Equations. Furtheremore, we give new numerical approximation for functions using mathematica coding called conformable fractional Fourier series approximation.

Key-Words: Fractional fourier series, Conformable fractional derivative.

Received: March 6, 2021. Revised: August 1, 2021. Accepted: August 30, 2021. Published: September 16, 2021.

\section{Introduction}

Fractional Fourier transform is one of the most important tools in applied sciences [1]. It has been proved that we can solve partial differential equations using Fractional Fourier transform [2]. Fractional partial differential equations also appeared to have many applications in physics and engineering. See [1], [3], [4], [5], [6], [7] and [8]. There are many definitions of fractional derivative. One of the most recent ones is the conformable fractional derivative [9].

For more applications on conformable fractional derivative we refer the reader to [10], [11], [12], [13], [14] and [15].

Recently [16], fractional Taylor power series was introduced, and a beautiful theory was layed there. However, no work is done on fractional Fourier series, though there is some work on fractional fourier transform.

The aim of this paper is to introduce conformable fractional Fourier series with separation of variables as a new technique to help us solve some fractional differential equations (Benjamin Bana Mahony and Heat Equations). These equations can not be solved using classical methods. Moreover, we create a new numerical approximation for functions using the mathematica coding called conformable fractional Fourier series approximation.

\section{Basics of Conformable Fractional Derivative}

A definition called conformable fractional derivative was introduced.

Definition 1 Let $\alpha>0$, then we define the fractional derivative of $f$ of order $\alpha$ as

$$
T_{\alpha}(f)(t)=\lim _{\epsilon \rightarrow 0} \frac{f\left(t+\epsilon t^{1-\alpha}\right)-f(t)}{\epsilon} .
$$

for all $t>0$ and $\alpha \in(0,1)$. We shall write $f^{\alpha}(t)$ for $T_{\alpha}(f)(t)$.

One can easily show that $T_{\alpha}$ satisfies all the properties in the following theorem.

Theorem 2 Let $\alpha \in(0,1]$ and $f, g$ be $\alpha$-differentiable at a point $t>0$. Then

(1) $T_{\alpha}(a f+b g)=a T_{\alpha}(f)+b T_{\alpha}(g)$, for all $a, b \in R$.

(2) $T_{\alpha}\left(t^{p}\right)=p t^{p-\alpha}$, for all $p \in R$.

(3) $T_{\alpha}(\lambda)=0$, for all constant functions $f(t)=\lambda$.

(4) $T_{\alpha}(f g)=g T_{\alpha}(f)+f T_{\alpha}(g)$.

(5) $T_{\alpha}\left(\frac{f}{g}\right)=\frac{g T_{\alpha}(f)-f T_{\alpha}(g)}{g^{2}}$

(6) In addition, if $f$ is differentiable, then $T_{\alpha}(f)(t)=t^{1-\alpha} \frac{d f}{d t}(t)$.

The conformable fractional derivatives of certain functions:

(1) $T_{\alpha}\left(\sin \frac{1}{\alpha} t^{\alpha}\right)=\cos \frac{1}{\alpha} t^{\alpha}$.

(2) $T_{\alpha}\left(\cos \frac{1}{\alpha} t^{\alpha}\right)=-\sin \frac{1}{\alpha} t^{\alpha}$. 
(3) $T_{\alpha}\left(e^{\frac{1}{\alpha} t^{\alpha}}\right)=e^{\frac{1}{\alpha} t^{\alpha}}$.

(4) $T_{\alpha}\left(t^{p}\right)=p t^{p-\alpha}$, for all $p \in R$.

On letting $\alpha=1$ in these derivatives, we get the corresponding ordinary derivatives.

One should notice that a function could be $\alpha$-differentiable at a point but not differentiable, for example, take $f(t)=2 \sqrt{t}$.

Then $T_{\frac{1}{2}}(f)(0)=\lim _{t \rightarrow 0^{+}} T_{\frac{1}{2}}(f)(t)=1$.

Where $T_{\frac{1}{2}}(f)(t)=1$, for $t>0$.

This is not the case for the known classical fractional derivatives since $T_{1}(f)(0)$ does not exist.

\section{Fractional Fourier Series}

Let $0<\alpha \leq 1$, and $\varphi:[0, \infty) \rightarrow R$ be defined by $\varphi(t)=\frac{t^{\alpha}}{\alpha}$ and $g:[0, \infty) \rightarrow R$ be any function.

Let $f:[0, \infty) \rightarrow R$ be defined by $f(t)=g(\varphi(t))$.

For example, if $g(t)=\cos t$, then $f(t)=\cos \left(\frac{t^{\alpha}}{\alpha}\right)$.

Definition 3 A function $f(t)$ is called $\alpha$-periodical with period $p$ if

$f(t)=g(\varphi(t))=g\left(\varphi(t)+\frac{p^{\alpha}}{\alpha}\right)$, for all $t \in[0, \infty)$.

As an example, $f(t)=\cos \left(\frac{t^{\alpha}}{\alpha}\right)$ is $\alpha$-periodic with period $p=(2 \alpha \pi)^{\frac{1}{\alpha}}$.

Definition 4 Two functions $f, h$ are called $\alpha$-orthogonal on $[0, b]$ if

$$
\int_{0}^{b} \frac{f(t) h(t)}{t^{1-\alpha}} d t=0
$$

Example $5 \cos \left(\frac{t^{\alpha}}{\alpha}\right)$ and $\cos \left(2 \frac{t^{\alpha}}{\alpha}\right)$ are $\alpha$-orthogonal on $\left[0,(\alpha 2 \pi)^{\frac{1}{\alpha}}\right]$.

Proof 6 Put the variable change $\frac{t^{\alpha}}{\alpha}=x$ and use property (6) in theorem 2.

We get $d x=t^{\alpha-1} d t=\frac{d t}{t^{1-\alpha}}$.

Further, when $t=0, x=0$, and when $t=(a 2 \pi)^{\frac{1}{\alpha}}$, $x=2 \pi$.

Hence

$$
\begin{gathered}
I=\int_{0}^{(a 2 \pi)^{\frac{1}{a}}} \cos \left(\frac{t^{a}}{a}\right) \cos \left(2 \frac{t^{a}}{a}\right) \frac{1}{t^{1-a}} d t \\
I=\int_{0}^{2 \pi} \cos (x) \cos (2 x) d x .
\end{gathered}
$$

Using the fact that $\cos (2 x)=1-2 \sin ^{2}(x)$ and the variable change $u=\sin (x)$, then $d u=\cos (x) d x$, when $x=0, u=0$ and when $x=2 \pi, u=0$.

Then the integral I became:

$$
I=\int_{0}^{0}\left(1-2 u^{2}\right) d u=0 .
$$

Hence a result as required.

In general, using the idea in Example 5; one can easily prove:

\section{Theorem 7}

(1) $\cos \left(n \frac{t^{\alpha}}{\alpha}\right)$ and $\cos \left(m \frac{t^{\alpha}}{\alpha}\right)$ are orthogonal on $\left[0,(\alpha 2 \pi)^{\frac{1}{\alpha}}\right]$, for all $n \neq m$.

(2) $\sin \left(n \frac{t^{\alpha}}{\alpha}\right)$ and $\sin \left(m \frac{t^{\alpha}}{\alpha}\right)$ are orthogonal on $\left[0,(\alpha 2 \pi)^{\frac{1}{\alpha}}\right]$, for all $n \neq m$.

(3) $\sin \left(n \frac{t^{\alpha}}{\alpha}\right)$ and $\cos \left(m \frac{t^{\alpha}}{\alpha}\right)$ are orthogonal on $\left[0,(\alpha 2 \pi)^{\frac{1}{\alpha}}\right]$, for all $n, m$.

Now, let us define the Fourier coefficients of an $\alpha$-periodic function with period $p$.

Definition 8 Let $f:[0, \infty) \rightarrow R$ be a given peicewise continuous $\alpha$-periodic with period p: Then we define:

(1) The cosine $\alpha$-Fourier coefficients of $f$ as

$$
a_{n}=\frac{2 \alpha}{p^{\alpha}} \int_{0}^{p} f(t) \cos \left(n \frac{t^{\alpha}}{\alpha}\right) \frac{d t}{t^{1-\alpha}}, n=0,1,2, \ldots
$$

(2) The sine $\alpha$-Fourier coefficients of $f$ as

$$
b_{n}=\frac{2 \alpha}{p^{\alpha}} \int_{0}^{p} f(t) \sin \left(n \frac{t^{\alpha}}{\alpha}\right) \frac{d t}{t^{1-\alpha}}, n=1,2,3, \ldots
$$

Example 9 Let $f(t)=\cos 2 \sqrt{t}$.

The cosine and sine $\frac{1}{2}$-Fourier coefficients of the function $f(t)$ is:

$$
a_{1}=1, \text { and } a_{n}=0 \text { for all } n \neq 1
$$

and

$$
b_{n}=0, \forall n \in N
$$

where

$$
p=(\alpha 2 \pi)^{\frac{1}{\alpha}} \text { and } \alpha=\frac{1}{2} .
$$

Now, we give the definition of the fractional Fourier series:

Definition 10 Let $f:[0, \infty) \rightarrow R$ be a given peicewise continuous function which is $\alpha$-periodical with period p: Then the $\alpha$-fractional Fourier series of $f$ associated with the interval $[0, p]$ is

$$
S(f)(t)=\frac{a_{0}}{2}+\sum_{n=1}^{\infty} a_{n} \cos \left(n \frac{t^{\alpha}}{\alpha}\right)+b_{n} \sin \left(n \frac{t^{\alpha}}{\alpha}\right)
$$

where $a_{n}$ and $b_{n}$ are as in above. 
Theorem 11 The fractional Fourier series of a piece wise continuous $\alpha$-periodical function converges pointwise to the average limit of the function at each point of discontinuity, and to the function at each point of continuity.

Proof 12 One can easily prove the previous classical results. See [10].

Example 13 Let $f(t)=2 \sqrt{t}$ if $0 \leq t \leq\left(\frac{\pi}{2}\right)^{2}$

and $f(t)=2 \sqrt{t}-2 \pi$ if $\left(\frac{\pi}{2}\right)^{2}<t \leq \pi^{2}$.

and $\alpha=\frac{1}{2}$ with $p=\pi^{2}$ on the interval $\left[0, \pi^{2}\right]$.

Then,

$$
\begin{aligned}
a_{0} & =\frac{\alpha}{p^{\alpha}} \int_{0}^{p} f(t) \frac{d t}{t^{1-\alpha}}=\frac{1}{2 \pi} \int_{0}^{\pi^{2}} f(t) \frac{d t}{\sqrt{t}} \\
& =\frac{1}{2 \pi}\left(\int_{0}^{\left(\frac{\pi}{2}\right)^{2}} 2 \sqrt{t} \frac{d t}{\sqrt{t}}+\int_{\left(\frac{\pi}{2}\right)^{2}}^{\pi^{2}}(2 \sqrt{t}-2 \pi) \frac{d t}{\sqrt{t}}\right) \\
& =0 .
\end{aligned}
$$

On the other hand we have :

$$
\begin{aligned}
a_{n} & =\frac{2 \alpha}{p^{\alpha}} \int_{0}^{p} f(t) \cos \left(n \frac{t^{\alpha}}{\alpha}\right) \frac{d t}{t^{1-\alpha}} \\
& \left.=\frac{1}{\pi} \int_{0}^{\pi^{2}} f(t) \cos (n 2 \sqrt{t})\right) \frac{d t}{\sqrt{t}} \\
& =\frac{1}{\pi} \int_{0}^{\left(\frac{\pi}{2}\right)^{2}} 2 \sqrt{t} \cos (n 2 \sqrt{t}) \frac{d t}{\sqrt{t}} \\
& +\frac{1}{\pi} \int_{\left(\frac{\pi}{2}\right)^{2}}^{\pi^{2}}(2 \sqrt{t}-2 \pi) \cos (n 2 \sqrt{t}) \frac{d t}{\sqrt{t}}
\end{aligned}
$$

Using change of variables: $\theta=2 \sqrt{t}$, we get $d \theta=\frac{d t}{\sqrt{t}}$. Observe that $\theta=0$ if $t=0, \theta=\pi$ if $t=\left(\frac{\pi}{2}\right)^{2}$, and $\theta=2 \pi$ if $t=\pi^{2}$, and using integration by parts.

Hence, the integral becomes :

$a_{n}=\frac{1}{\pi} \int_{0}^{\pi} \theta \cos (n \theta) d \theta+\frac{1}{\pi} \int_{\pi}^{2 \pi}(\theta-2 \pi) \cos (n \theta) d \theta=0$.

Similarly we get

$$
b_{n}=\frac{2 \alpha}{p^{\alpha}} \int_{0}^{p} f(t) \sin \left(n \frac{t^{\alpha}}{\alpha}\right) \frac{d t}{t^{1-\alpha}}=\frac{2(-1)^{n+1}}{n} .
$$

So

$$
\begin{aligned}
S_{\alpha}(f)(t) & =\frac{a_{0}}{2}+\sum_{n=1}^{\infty} a_{n} \cos \left(n \frac{t^{\alpha}}{\alpha}\right)+b_{n} \sin \left(n \frac{t^{\alpha}}{\alpha}\right) \\
& =\sum_{n=1}^{\infty} \frac{2(-1)^{n+1}}{n} \sin \left(n \frac{t^{\alpha}}{\alpha}\right) .
\end{aligned}
$$

The figures below represent the function alongside its $\alpha$-Fractional Fourier series approximation for 10, 100, 10000 terms where $\alpha=\frac{1}{2}, \alpha=\frac{1}{4}$ and $\alpha=1$ (classical Fourier series) respectively.

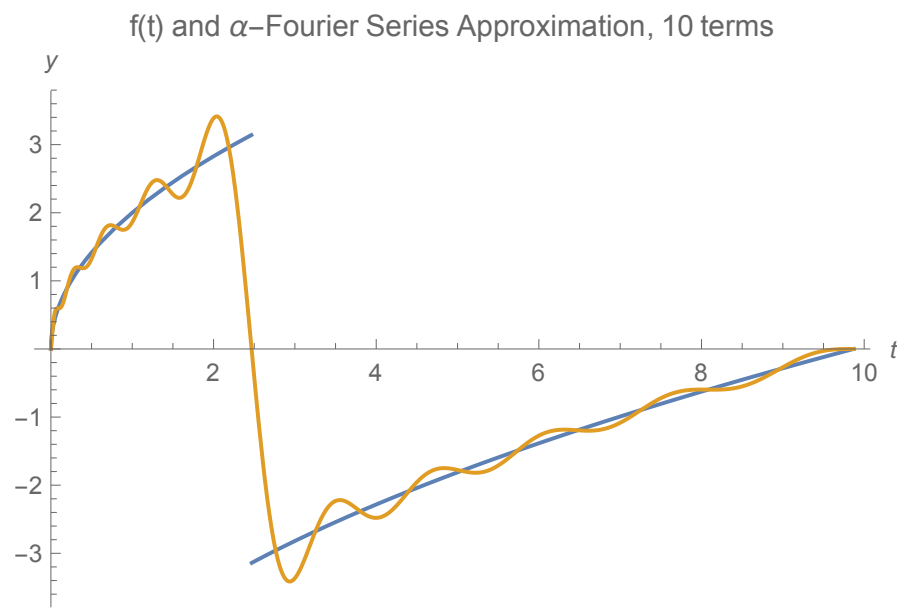

Figure 1: $f(t)$ and alpha-Fractional Fourier Series Approximation, 10 terms.

$f(t)$ and $\alpha$-Fourier Series Approximation, 100 terms

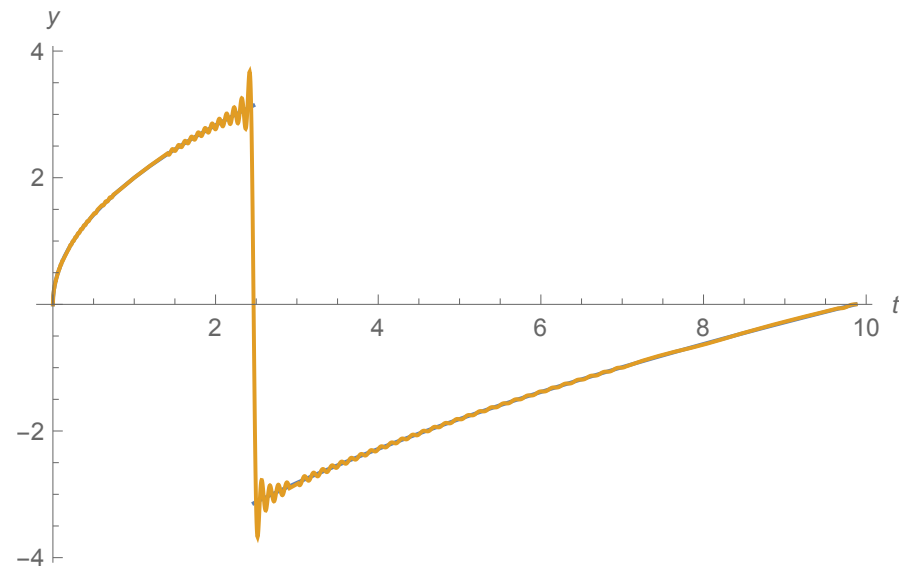

Figure 2: $f(t)$ and alpha-Fractional Fourier Series Approximation, 100 terms. 
$\mathrm{f}(\mathrm{t})$ and $\alpha$-Fourier Series Approximation, 10000 terms

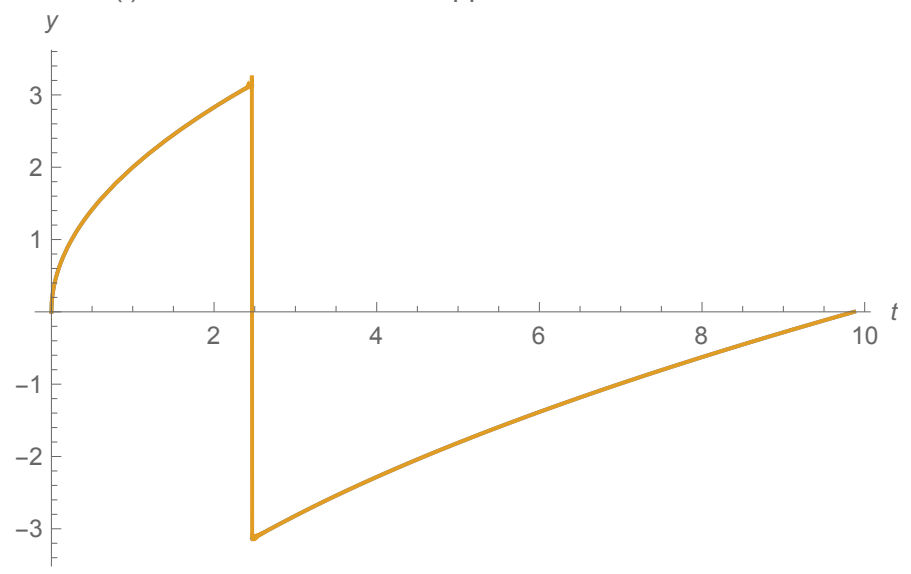

Figure 3: $f(t)$ and alpha-Fractional Fourier Series Approximation, 10000 terms.

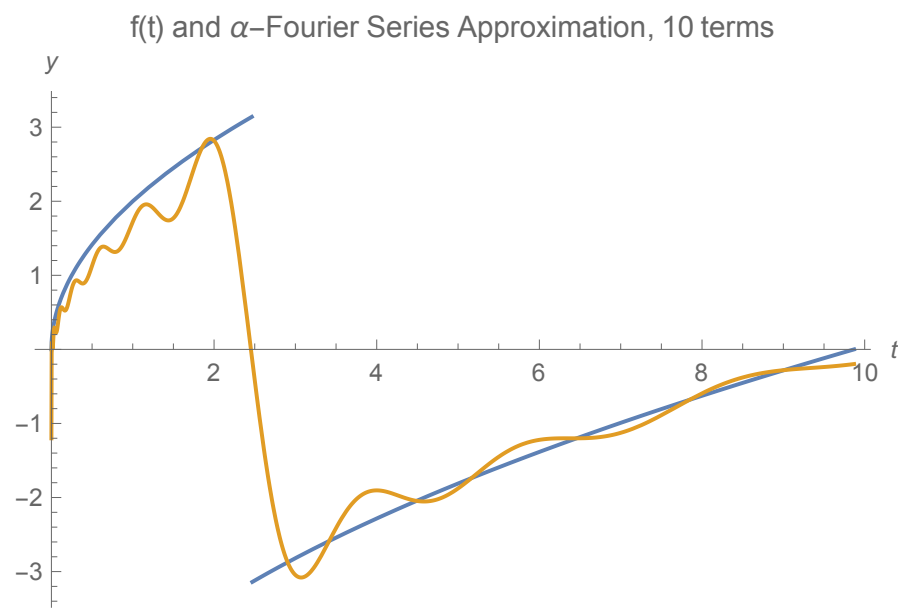

Figure 4: $f(t)$ and alpha-Fractional Fourier Series Approximation, 10 terms.

$\mathrm{f}(\mathrm{t})$ and $\alpha$-Fourier Series Approximation, 100 terms

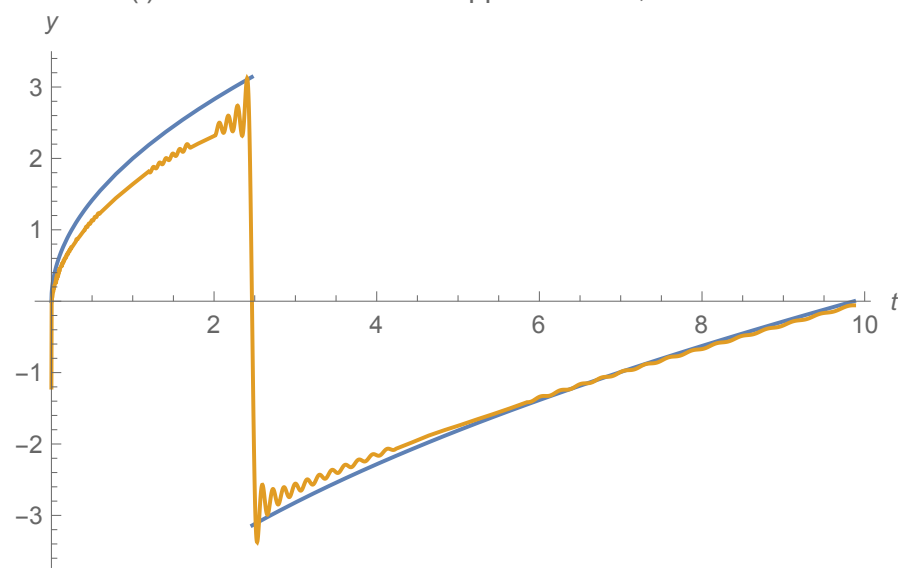

Figure 5: $f(t)$ and alpha-Fractional Fourier Series Approximation, 100 terms. $\mathrm{f}(\mathrm{t})$ and $\alpha$-Fourier Series Approximation, 10000 terms

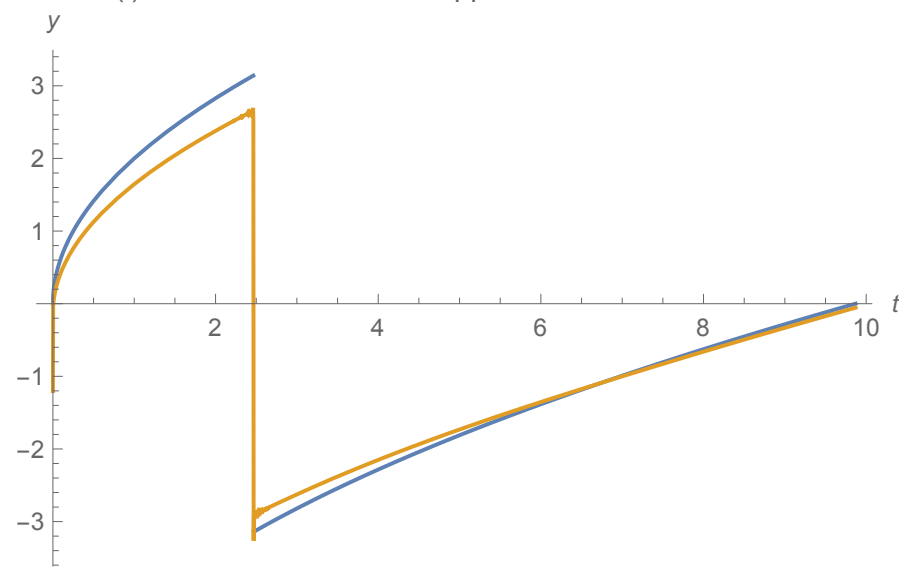

Figure 6: $f(t)$ and alpha-Fractional Fourier Series Approximation, 10000 terms.

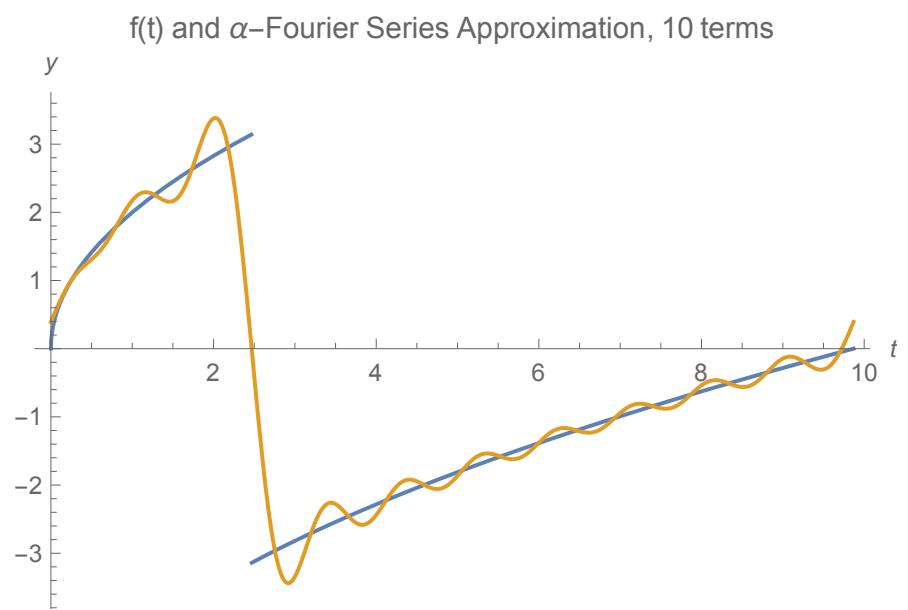

Figure 7: $f(t)$ and Fourier Series Approximation, 10 terms.

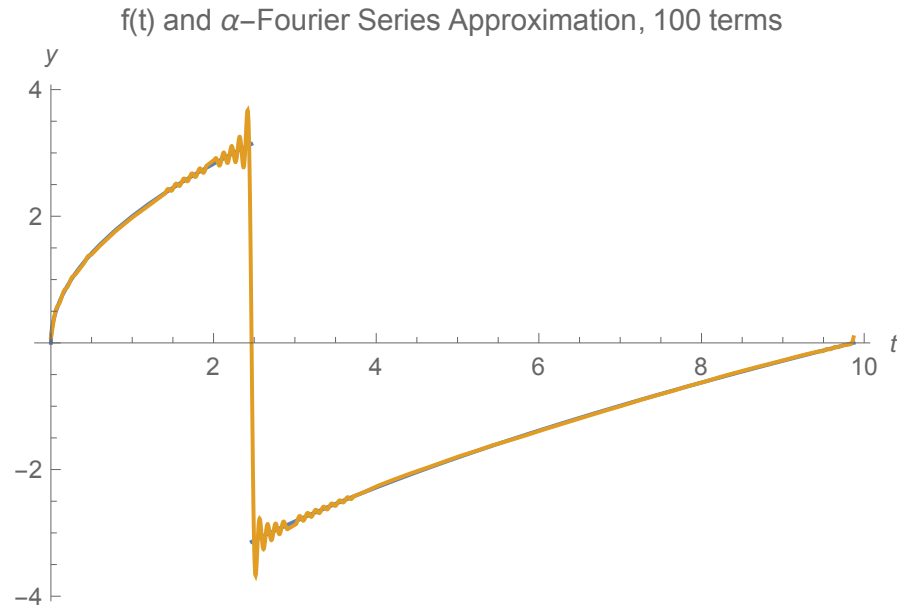

Figure 8: $f(t)$ and Fourier Series Approximation, 100 terms. 
$\mathrm{f}(\mathrm{t})$ and $\alpha$-Fourier Series Approximation, 10000 terms

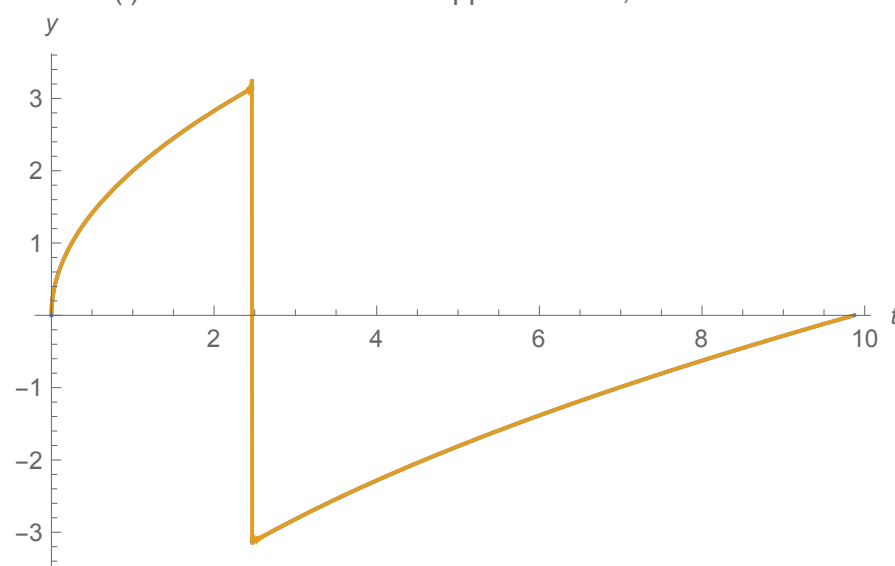

Figure 9: $f(t)$ and Fourier Series Approximation, 10000 terms.

\section{Solution of Fractional Benjamin Bana Mahony Equation}

We will attempt to solve an equation called Fractional Benjamin Bana Mahony Equation using separation of variables and fractional Fourier series :

$$
D_{t}^{\beta} D_{t}^{\beta} U+D_{x}^{\alpha} U=D_{t}^{\beta} D_{t}^{\beta} D_{x}^{\alpha} D_{x}^{\alpha} U .0<\alpha<1
$$

subject to the conditions :

$$
\begin{gathered}
U(0, t)=0 ; \quad U_{x}^{\alpha}(1, t)=t^{2 \beta} ; \quad x>0 . \\
U(x, 0)=0 ; \quad U(x, 1)=0 ; \quad 1>t>0 .
\end{gathered}
$$

\section{Solution :}

We will be using separation of variables technique.

Let

$$
U(x, t)=P(x) Q(t) .
$$

Substitute in equation (1) to get :

$$
P(x) Q^{2 \beta}(t)+P^{\alpha}(x) Q(t)=P^{2 \alpha}(x) Q^{2 \beta}(t) .
$$

Here $P(x)$ and $Q(t)$ are the unknowns.

From which we get :

$$
\frac{Q^{2 \beta}(t)}{Q(t)}=\frac{P^{\alpha}(x)}{P^{2 \alpha}(x)-P(x)} .
$$

Since $x$ and $t$ are independent variables; then we get :

$$
\frac{Q^{2 \beta}(t)}{Q(t)}=\frac{P^{\alpha}(x)}{P^{2 \alpha}(x)-P(x)}=\lambda .
$$

where $\lambda$ is a constant to be determined. Hence we obtain :

$$
Q^{2 \beta}(t)-\lambda Q(t)=0 .
$$

$$
\lambda P^{2 \alpha}(x)-P^{\alpha}(x)-\lambda P(x)=0 .
$$

Conditions suggest that we work with equation (3) first.

Then the auxiliary equation of equation (3) is

$$
r^{2}-\lambda=0 .
$$

There are three possibilities for $\lambda$ :

Case (i) : $\lambda=0$ :

then equation (3) becomes :

$$
Q^{2 \beta}(t)=0 .
$$

We get

$$
Q(t)=c_{1}+c_{2} \frac{t^{\beta}}{\beta}
$$

for some constants $c_{1}$ and $c_{2}$.

Conditions implies that : $c_{1}=c_{2}=0$. So

$$
Q(t)=0 .
$$

Hence, no non trivial solution exists when $\lambda=0$.

Case (ii) : $\lambda=\mu^{2}>0$ :

then equation (3) becomes :

$$
Q^{2 \beta}(t)-\mu^{2} Q(t)=0 .
$$

So the characteristic equation of equation (3) becomes $r^{2}-\mu^{2}=0$, we get two distinct solution $r_{1}=\mu$, $r_{2}=-\mu$.

Therefore the solution has the form

$$
Q(t)=c_{1} e^{\mu \frac{t^{\beta}}{\beta}}+c_{2} e^{-\mu \frac{t^{\beta}}{\beta}}
$$

for some constants $c_{1}$ and $c_{2}$.

But again conditions show that $Q(0)=c_{1}+c_{2}=0$, $c_{1}=-c_{2}$, also $Q(1)=c_{1} e^{\frac{\mu}{\beta}}+c_{2} e^{\frac{-\mu}{\beta}}=0$.

So, $c_{1}\left(e^{\frac{\mu}{\beta}}-e^{-\frac{\mu}{\beta}}\right)=0$, thus $c_{1}=c_{2}=0$.

Hence, no non trivial solution exists when $\lambda=\mu^{2}$.

Case (iii) : $\lambda=-\mu^{2}<0$ :

then equation (3) becomes :

$$
Q^{2 \beta}(t)+\mu^{2} Q(t)=0 .
$$

Then the auxiliary equation of equation (3) becomes $r^{2}+\mu^{2}=0$, we get two distinct complex solution $r_{1}=i \mu, r_{2}=-i \mu$, and since the real part in $r_{1}$ and $r_{2}$ equal 0 .

Therefore the solution is given as follows :

$$
Q(t)=c_{1} \sin \left(\mu \frac{t^{\beta}}{\beta}\right)+c_{2} \cos \left(\mu \frac{t^{\beta}}{\beta}\right) .
$$


for some constants $c_{1}$ and $c_{2}$.

Using conditions to get :

$$
Q(0)=c_{2}=0 ; \quad Q(1)=c_{1} \sin \frac{\mu}{\beta}=0 .
$$

Thus

$$
\sin \frac{\mu}{\beta}=0, \text { so } \frac{\mu}{\beta}=n \pi \text {, ie } \mu=n \pi \beta
$$

where $n=1,2,3, \ldots$

Hence

$$
Q_{n}(t)=c_{n} \sin \left(n \pi t^{\beta}\right) .
$$

Now, we go back to equation (4) we find :

$$
-n^{2} \pi^{2} \beta^{2} P^{2 \alpha}(x)-P^{\alpha}(x)+n^{2} \pi^{2} \beta^{2} P(x)=0 .
$$

The auxiliary equation of equation (6) is :

$$
-n^{2} \pi^{2} \beta^{2} r^{2}-r+n^{2} \pi^{2} \beta^{2}=0 .
$$

Thus

$$
\Delta=1+4 n^{4} \pi^{4} \beta^{4}>0 .
$$

So

$$
r_{1}=\frac{-1+\sqrt{\Delta}}{2 n^{2} \pi^{2} \beta^{2}} \text { and } r_{2}=\frac{-1-\sqrt{\Delta}}{2 n^{2} \pi^{2} \beta^{2}} .
$$

Hence the solution of this equation is :

$$
P(x)=k_{1} e^{r_{1} \frac{x^{\alpha}}{\alpha}}+k_{2} e^{r_{2} \frac{x^{\alpha}}{\alpha}} .
$$

for some constants $k_{1}$ and $k_{2}$.

Conditions implies that :

$$
\begin{gathered}
P(0)=k_{1}+k_{2}=0, \text { so } k_{2}=-k_{1}, \text { thus } \\
P(x)=k_{1}\left(e^{r_{1} \frac{x^{\alpha}}{\alpha}}-e^{r_{2} \frac{x^{\alpha}}{\alpha}}\right) .
\end{gathered}
$$

Hence

$$
P_{n}(x)=k_{n}\left(e^{r_{1} \frac{x^{\alpha}}{\alpha}}-e^{r_{2} \frac{x^{\alpha}}{\alpha}}\right) .
$$

Combining (5) and (7) to get :

$$
U(x, t)=\sum_{n=1}^{\infty} b_{n}\left(e^{r_{1} \frac{x^{\alpha}}{\alpha}}-e^{r_{2} \frac{x^{\alpha}}{\alpha}}\right) \sin \left(n \pi t^{\beta}\right) .
$$

Conditions show that :

$$
t^{2 \beta}=\sum_{n=1}^{\infty} b_{n}\left(r_{1} e^{r_{1} \frac{1}{\alpha}}-r_{2} e^{r_{2} \frac{1}{\alpha}}\right) \sin \left(n \pi t^{\beta}\right) .
$$

Using the $\beta$-Fractional Fourier series of $t^{2 \beta}$, we find that :

$$
\begin{aligned}
b_{n} & =\frac{2 \beta}{p^{\beta}} \int_{0}^{p} t^{2 \beta} \sin \left(n \pi t^{\beta}\right) \frac{d t}{t^{1-\beta}} \\
& =\frac{2 \beta}{p^{\beta}} \int_{0}^{p} t^{3 \beta-1} \sin \left(n \pi t^{\beta}\right) d t
\end{aligned}
$$

Now we have to use this variable change $u=t^{\beta}$ and $d u=\beta t^{\beta-1}$ to get :

$$
\begin{aligned}
b_{n} & =\frac{2 \beta}{p^{\beta}} \int_{0}^{p^{\beta}} t^{3 \beta-1} \sin (n \pi u) \frac{t^{1-\beta}}{\beta} d u \\
& =\frac{2}{p^{\beta}} \int_{0}^{p^{\beta}} u^{2} \sin (n \pi u) d u .
\end{aligned}
$$

Then :

$$
b_{n}=\frac{2}{p^{\beta}}\left[-u^{2} \frac{\cos (n \pi u)}{n \pi}+2 u \frac{\sin (n \pi u)}{n^{2} \pi^{2}}+2 \frac{\cos (n \pi u)}{n^{3} \pi^{3}}\right]_{0}^{p^{\beta}} .
$$

Thus :

$b_{n}=-2 p^{\beta} \frac{\cos \left(n \pi p^{\beta}\right)}{n \pi}+4 \frac{\sin \left(n \pi p^{\beta}\right)}{n^{2} \pi^{2}}+4 \frac{\cos \left(n \pi p^{\beta}\right)}{p^{\beta} n^{3} \pi^{3}}-\frac{4}{p^{\beta} n^{3} \pi^{3}}$.

Therefore :

$$
U(x, t)=\sum_{n=1}^{\infty} b_{n}\left(e^{r_{1} \frac{x^{\alpha}}{\alpha}}-e^{r_{2} \frac{x^{\alpha}}{\alpha}}\right) \sin \left(n \pi \beta \frac{t^{\beta}}{\beta}\right) .
$$

Where :

$b_{n}=-2 p^{\beta} \frac{\cos \left(n \pi p^{\beta}\right)}{n \pi}+4 \frac{\sin \left(n \pi p^{\beta}\right)}{n^{2} \pi^{2}}+4 \frac{\cos \left(n \pi p^{\beta}\right)}{p^{\beta} n^{3} \pi^{3}}-\frac{4}{p^{\beta} n^{3} \pi^{3}}$.

\section{Application}

Now, as an application we will use fractional Fourier series to solve the Conformable Fractional heat partial differential equation as demonstrated in the example below.

\section{Example 14}

$$
\begin{aligned}
\frac{\partial^{\alpha}}{\partial t^{\alpha}}\left(\frac{\partial^{\alpha} u(x, t)}{\partial t^{\alpha}}\right) & =c^{2} \frac{\partial^{2} u(x, t)}{\partial x^{2}} \\
0<t & , \quad 0<x<L \\
u(0, t) & =0 \\
u(L, t) & =0 \\
\frac{\partial u}{\partial x}(0, t) & =t^{2 \alpha} \\
u(x, 0) & =0
\end{aligned}
$$

\section{Solution:}

Let us use separation of variables technique.

So let $u(x, t)=P(x) Q(t)$, substitute in the equation to get

$$
P(x) Q^{2 \alpha}(t)=c^{2} P^{2}(x) Q(t) .
$$

Since $x$ and t are independent variables, then we get

$$
\frac{Q^{2 \alpha}(t)}{Q(t)}=\frac{c^{2} P^{2}(x)}{P(x)}=\lambda .
$$


for some constant $\lambda$.

Consequently,

$$
\begin{aligned}
Q^{2 \alpha}(t)-\lambda Q(t) & =0 \\
c^{2} P^{2}(x)-\lambda P(x) & =0
\end{aligned}
$$

We start with equation $(7), P^{2}(x)-\lambda P(x)=0$. Then the auxiliary equation of equation (7) is $r^{2}-\frac{\lambda}{c^{2}}=0$. Now there are three possibilities for $\lambda$ :

Case (i) : $\lambda=0$. Then $r=0$, so the solution is $P(x)=c_{1}+c_{2} x$.

Using condition (2), we get $c_{1}=0$, again using condition (3), we get $c_{2}=0$.

So $P(x)=0$. Hence, no non trivial solution when $\lambda=0$.

Case (ii) : $\lambda=\mu^{2}>0$. So $r=+\frac{\mu}{\lambda}, r=-\frac{\mu}{\lambda}$, then the solution is $P(x)=c_{1} \sinh \frac{\mu}{c} x+c_{2} \cosh \frac{\mu}{c} x$.

Using condition (2) and (3), we get $c_{1}=0$ and $c_{2}=0$, so $P(x)=0$.

Finally, no non trivial solution when $\lambda>0$.

Case (iii) : $\lambda=-\mu^{2}<0$. so $r=+i \frac{\mu}{\lambda}, r=-i \frac{\mu}{\lambda}$, then the solution is $P(x)=c_{1} \sin \frac{\mu}{c} x+c_{2} \cos \frac{\mu}{c} x$.

Using condition (2), we get $c_{2}=0$, also condition (3), we get

$P(L)=c_{1} \sin \frac{\mu}{c} L=0$, so $\sin \frac{\mu}{c} L=0$.

Hence, $\mu=\frac{c}{L} n \pi, n=1,2,3, \ldots$. which gives that $\lambda=-\left(\frac{c}{L} n \pi\right)^{2}, n=1,2,3, \ldots$

Thus

$$
P_{n}(x)=c_{n} \sin \left(\frac{n \pi}{L} x\right)
$$

Now, we return back to equation (6),

$$
Q^{2 \alpha}(t)-\lambda Q(t)=0
$$

So

$$
Q^{2 \alpha}(t)+\left(\frac{c}{L} n \pi\right)^{2} Q(t)=0 .
$$

Its auxiliary equation is $r^{2}+\left(\frac{c}{L} n \pi\right)^{2}=0$, thus we get two distinct complex solution

$$
r_{1}=i \frac{c}{L} n \pi, r_{2}=-i \frac{c}{L} n \pi
$$

and since the real part equal 0 , the solution is given as follow:

$$
Q(t)=b_{1} \cos \left(\frac{c}{L} n \pi \frac{t^{\alpha}}{\alpha}\right)+b_{2} \sin \left(\frac{c}{L} n \pi \frac{t^{\alpha}}{\alpha}\right) .
$$

Condition (5) implies that $b_{1}=0$.

So

$$
Q(t)=b_{2} \sin \left(\mu \frac{t^{\alpha}}{\alpha}\right) .
$$

Hence,

$$
Q_{n}(t)=b_{n} \sin \left(n \pi \frac{c}{L} \frac{t^{\alpha}}{\alpha}\right)
$$

Combining (8) and (9) to get

$$
u(x, t)=\sum_{n=1}^{\infty} a_{n} \sin \left(\frac{n \pi}{L} x\right) \sin \left(n \pi \frac{c}{L} \frac{t^{\alpha}}{\alpha}\right) .
$$

Now, using condition (4), $\frac{\partial u}{\partial x}(0, t)=t^{2 \alpha}$, to get

$$
t^{2 \alpha}=\sum_{n=1}^{\infty} a_{n} \frac{n \pi}{L} \sin \left(n \pi \frac{c}{L} \frac{t^{\alpha}}{\alpha}\right) .
$$

Using the $\alpha$-Fractional Fourier series of $t^{2 \alpha}$, we find that

$$
\begin{aligned}
a_{n} & =\frac{2 \alpha}{p^{\alpha}} \int_{0}^{p} t^{2 \alpha} \sin \left(n \frac{t^{\alpha}}{\alpha}\right) \frac{d t}{t^{1-\alpha}} \\
& =\frac{2 \alpha}{p^{\alpha}} \int_{0}^{p} t^{3 \alpha-1} \sin \left(n \frac{t^{\alpha}}{\alpha}\right) d t .
\end{aligned}
$$

By using the substitution $u=t^{\alpha}$, and $d u=\alpha t^{\alpha-1} d t$, then

$$
\begin{aligned}
a_{n} & =\frac{2 \alpha}{p^{\alpha}} \int_{0}^{p^{\alpha}} t^{3 \alpha-1} \sin \left(n \frac{u}{\alpha}\right) \frac{t^{1-\alpha}}{\alpha} d u \\
& =\frac{2}{p^{\alpha}} \int_{0}^{p^{\alpha}} t^{2 \alpha} \sin \left(n \frac{u}{\alpha}\right) d u \\
& =\frac{2}{p^{\alpha}} \int_{0}^{p^{\alpha}} u^{2} \sin \left(n \frac{u}{\alpha}\right) d u .
\end{aligned}
$$

\section{Consequently}

$$
\begin{aligned}
a_{n} & =\frac{2}{p^{\alpha}}\left[-u^{2} \cos \left(\frac{n}{\alpha} u\right) \cdot \frac{\alpha}{n}+2 u \sin \left(\frac{n}{\alpha} u\right) \cdot \frac{\alpha^{2}}{n^{2}}\right]_{0}^{p^{\alpha}} \\
& +\frac{2}{p^{\alpha}}\left[2 \cos \left(\frac{n}{\alpha} u\right) \cdot \frac{\alpha^{3}}{n^{3}}\right]_{0}^{p^{\alpha}} \\
& =\frac{2}{p^{\alpha}}\left[-p^{2 \alpha} \cos \left(\frac{n}{\alpha} p^{\alpha}\right) \cdot \frac{\alpha}{n}+2 p^{\alpha} \sin \left(\frac{n}{\alpha} p^{\alpha}\right) \cdot \frac{\alpha^{2}}{n^{2}}\right] \\
& +\frac{2}{p^{\alpha}}\left[2 \cos \left(\frac{n}{\alpha} p^{\alpha}\right) \cdot \frac{\alpha^{3}}{n^{3}}\right]-2 \frac{\alpha^{3}}{n^{3}} \\
& =-2 p^{\alpha} \cos \left(\frac{n}{\alpha} p^{\alpha}\right) \cdot \frac{\alpha}{n}+4 \sin \left(\frac{n}{\alpha} p^{\alpha}\right) \cdot \frac{\alpha^{2}}{n^{2}} \\
& +\frac{4}{p^{\alpha}} \cos \left(\frac{n}{\alpha} p^{\alpha}\right) \cdot \frac{\alpha^{3}}{n^{3}}-2 \frac{\alpha^{3}}{n^{3}} .
\end{aligned}
$$

Therefore

$$
u(x, t)=\sum_{n=1}^{\infty} a_{n} \sin \left(\frac{n \pi}{L} x\right) \sin \left(n \pi \frac{c}{L} \frac{t^{\alpha}}{\alpha}\right) .
$$


Where

$$
\begin{aligned}
a_{n} & =-2 p^{\alpha} \cos \left(\frac{n}{\alpha} p^{\alpha}\right) \cdot \frac{\alpha}{n}+4 \sin \left(\frac{n}{\alpha} p^{\alpha}\right) \cdot \frac{\alpha^{2}}{n^{2}} \\
& +\frac{4}{p^{\alpha}} \cos \left(\frac{n}{\alpha} p^{\alpha}\right) \cdot \frac{\alpha^{3}}{n^{3}}-2 \frac{\alpha^{3}}{n^{3}} .
\end{aligned}
$$

IN our paper, we use the mathematica coding programm to see the $\alpha$-Fractional Fourier series approximation and the classical Fourier series approximation of functions.

The figures bellow represent the solution of Conformable Fractional heat partial differential equation with three different terms.

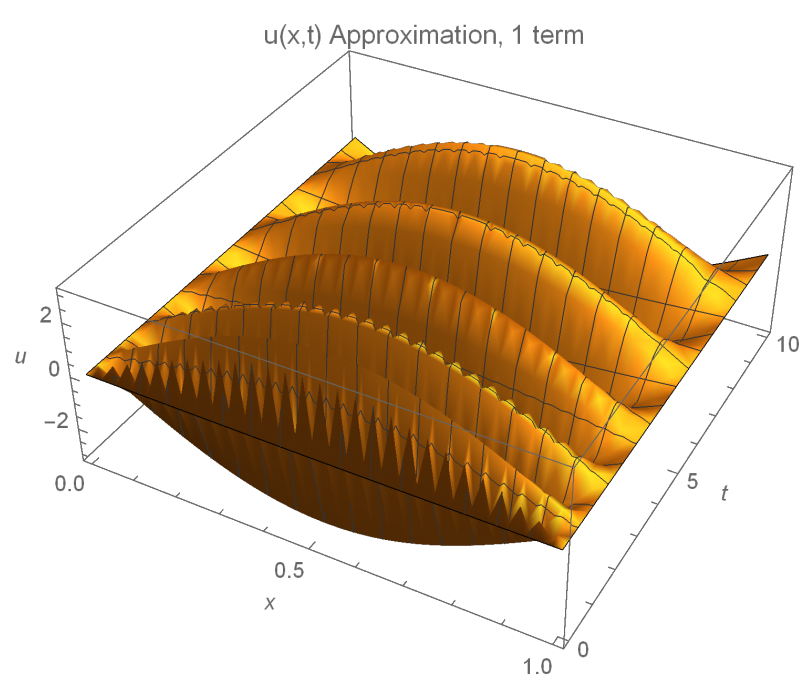

Figure 10: $\mathrm{u}(\mathrm{x}, \mathrm{t})$ Approximation, 1 term.

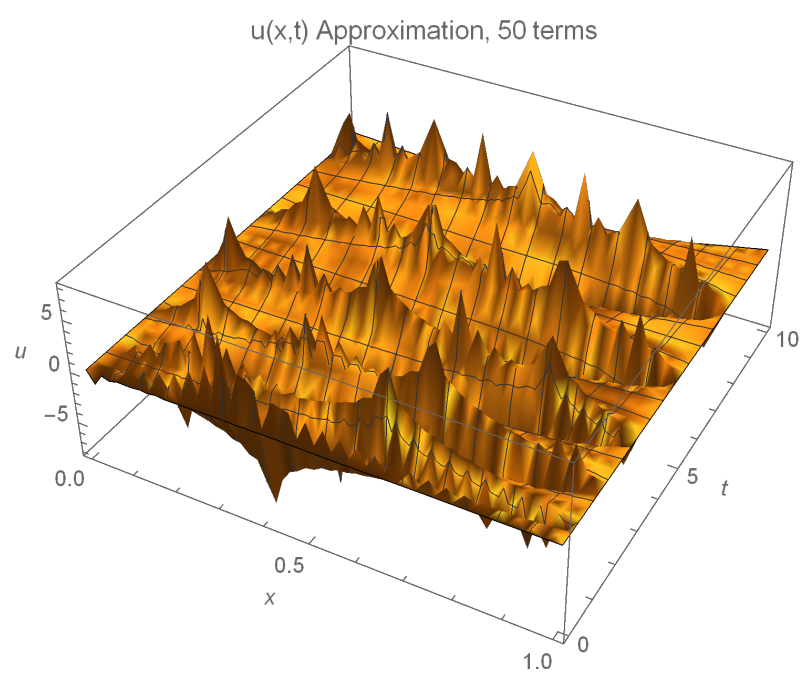

Figure 11: $\mathrm{u}(\mathrm{x}, \mathrm{t})$ Approximation, 50 terms.

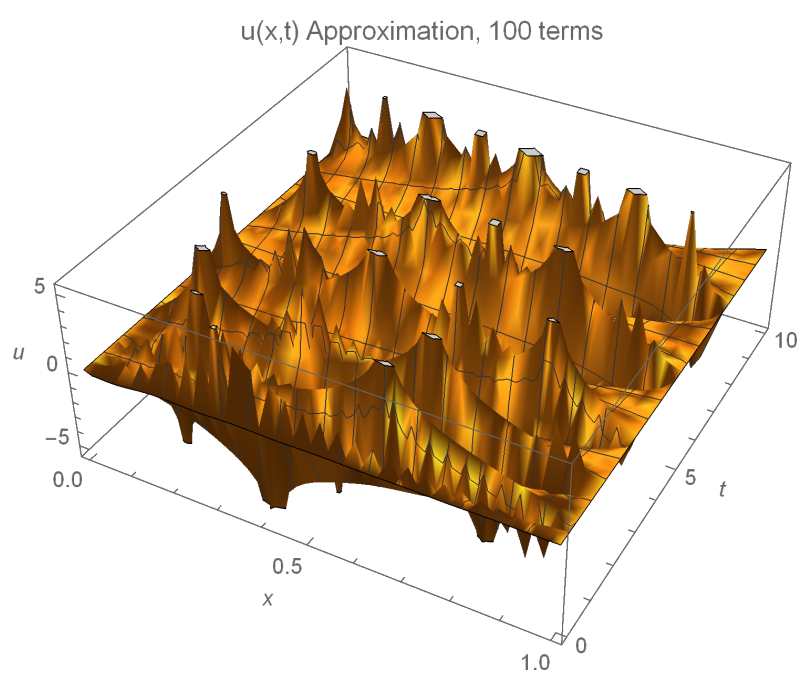

Figure 12: $\mathrm{u}(\mathrm{x}, \mathrm{t})$ Approximation, 100 terms.

\section{Conclusion}

The results explained in the previous sections show that the separation variables with fractional Fourier series technique solves some difficult problems that cannot be solved with classical methods, also we can use the conformable fractional Fourier series approximation to approach some functions.

\section{Closing remark:}

(i) In the Example 11, $\alpha=\frac{1}{2}$ give best approximation of the function $f(t)$ then $\alpha=\frac{1}{4}$.

(ii) Is the $\alpha$-Fractional Fourier series give best approximation of functions then the classical Fourier series and vice versa?

(iii) What is the value of $\alpha$ which give the best and perfect approximation in $\alpha$-Fractional Fourier series approximation? and how to fix it?

\section{References:}

[1] Haldun, M. Ozaktas and M. Alper Kutay. (), Introduction to the Fractional Fourier Transform and its Applications, TR-06533 Bilkent, Ankara, Turkey.

[2] Yong-Ju, Y., shun-Qin, W. (2015), Local Fractional Fourier Series Method for solving non Linear Equations with Local Fractional Operators, Journa of Mathematical Problems in Engeneering.

[3] Zhenyan, F., Jun Yu, Z., Xiaodong, Z., and Nikos E, M. (2019), Threshold Filtering for Phoneme Pronunciation Signals Based on FrFT. 
International Journal of Circuits, Systems and Signal Processing.

[4] J. F. Wang, H. Y. Xu. (2012), Speech Enhancement Based on FrFT Filtering. Computer Engineering and Applications, 48th ed., vol. 12. pp. 129-134.

[5] Y. H. Bu, P. B. Wang. (2016), Filtering Based on Fractional Fourier Transform, Ship Electronic Engineering, 36th ed., vol. 4. pp. 38-40.

[6] P. Kumar, S. Kansal. (2017), Noise Removal in Speech signal using Fractional Fourier Transform. IEEE International Conference on Information Communication, Instrumentation and Control Conference. India.

[7] J. Xia , J. Y. Zhang, X.B. Li. (2017), Weak signal detection based on FrFTdomain Adaptive Filtering Algorithm. Cie International Conference on Radar. IEEE. China.

[8] H. Li, Y.L. Qin, Y.P. Li, H.Q. Wang, X. Li. (2011), A FrFT Based Algorithm for Doppler Frequency Rate Estimation from LFM Coherent Pulse Train. Journal of Electronics and Information Technology, 11th ed., vol. 32. pp.27182723.

[9] Khalil, R., Al Horani, M., Yousef, A. and Sababheh, M. (2014), A new Denition of Fractional Derivative, J. Comput. Appl. Math. 264. pp. 6570.

[10] Abu Hammad, M., Khalil, R. (2014), Fractional Fourier Series with Applications, j.ajcam. 4(6): 187-191.

[11] Abu-Hammad, M., and Khalil, R. (2014), Legendre fractional differential equation and Legender fractional polynomials. I.J of Applied Mathematical Research, 3 (3) 214-219.

[12] Abu-Hammad, M., and Khalil, R. (2014) Abel., $S$ Formula And Wronskian For Conformable Fractional Differential Equations. I.J. Differential Equations and Applications, 13 177-183.

[13] Bouchenak, A., AlHorani, M., Khalil, R. (2021) Tensor product technique and atomic solution of fractional Bate Man Burger equation. J. math. Comput. Sci, 1927-5307.

[14] Khalil, R., Abu Hammad, M. (2014), Conformable fractional Heat differential equation, ijpam. v94i2.8.
[15] Z. Al-zhour, F. Alrawajeh, N. Al-mutairi and R. Alkhasawneh. (2019), New results on the conformable fractional Sumudu transforms:Theories and applications. International Journal of Analysis and Applications. 10.28924/2291-863.

[16] Abdeljawad, T. (2015), On conformable fractional calculus, J. Comput. Appl. Math. 279,5766 .

\section{Creative Commons Attribution License 4.0 (Attribution 4.0 International, CC BY 4.0)}

This article is published under the terms of the Creative Commons Attribution License 4.0 https://creativecommons.org/licenses/by/4.0/deed.en_US 